ARTICLE

DOI: $10.1038 /$ s41467-018-07145-6

\title{
Calcium stabilizes the strongest protein fold
}

\author{
Lukas F. Milles (1) ${ }^{1}$, Eduard M. Unterauer ${ }^{1}$, Thomas Nicolaus ${ }^{1} \&$ Hermann E. Gaub ${ }^{1}$
}

Staphylococcal pathogens adhere to their human targets with exceptional resilience to mechanical stress, some propagating force to the bacterium via small, lg-like folds called B domains. We examine the mechanical stability of these folds using atomic force microscopybased single-molecule force spectroscopy. The force required to unfold a single B domain is larger than $2 \mathrm{nN}$ - the highest mechanostability of a protein to date by a large margin. B domains coordinate three calcium ions, which we identify as crucial for their extreme mechanical strength. When calcium is removed through chelation, unfolding forces drop by a factor of four. Through systematic mutations in the calcium coordination sites we can tune the unfolding forces from over $2 \mathrm{nN}$ to $0.15 \mathrm{nN}$, and dissect the contribution of each ion to $\mathrm{B}$ domain mechanostability. Their extraordinary strength, rapid refolding and calcium-tunable force response make B domains interesting protein design targets.

\footnotetext{
${ }^{1}$ Lehrstuhl für Angewandte Physik and Center for Nanoscience, Ludwig-Maximilians-University, Amalienstr. 54, 80799 Munich, Germany. Correspondence and requests for materials should be addressed to L.F.M. (email: lukas.milles@physik.uni-muenchen.de) or to H.E.G. (email: gaub@lmu.de)
} 
P athogenic bacteria have evolved to strongly and persistently adhere to their hosts using a variety of mechanisms. Among them are microbial surface components recognizing adhesive matrix molecules (MSCRAMMs), which adhere to target proteins of their human hosts ${ }^{1,2}$. Covalently bound to the Grampositive peptidoglycan and extruding into the extracellular space, these adhesins target sequences on the order of 15 amino acids in human proteins, notably all chains of fibrinogen, but also other members of the extracellular and adhesive matrix, such as fibronectin ${ }^{3}$ and keratin ${ }^{4}$. Initial adhesion is crucial to begin infection, especially under hydrodynamic forces. The ligand binding region $\mathrm{A}$ at the $\mathrm{N}$-terminus of these adhesins employs the "Dock, Lock and Latch" (DLL) mechanism ${ }^{5,6}$. In DLL, the peptide target is tightly confined between the N2 and N3 domain of region $\mathrm{A}$, achieving mechanically hyperstable adhesion to host proteins. These adhesins, such as SdrG from S. epidermidis, and its homologs from S. aureus (ClfA, ClfB, Bbp, FnBPA, and SdrE) can withstand forces in the $2 \mathrm{nN}$ force regime ${ }^{7-12}$, approaching the strength of a covalent bond ${ }^{13}$. This extreme strength is achieved through a recently identified molecular mechanism ${ }^{9}$. Along their stalks are so-called B domains, which appear in adhesins such as S.aureus SdrD and Bbp, S. epidermidis SdrG, and S. saprophyticus $\mathrm{UafA}^{14}$. B domains are small domains $(\sim 13 \mathrm{kDa})$ that link the ligand binding region to a sortase motif, which mediates covalent anchoring of the adhesin to the bacterial peptidoglycan ${ }^{15}$. Thus, it is to be expected that these folds must propagate the extreme mechanical force withstood by the DLL adhesin.

Using atomic force microscopy-based (AFM) single-molecule force spectroscopy (SMFS) ${ }^{16-19}$ we investigate the mechanical strength of B domains from S. epidermidis $\mathrm{SdrG}^{20}$ and S. aureus $\mathrm{SdrD}^{21,22}$. We found their mechanostability to be exceptional, far exceeding all other proteins investigated to date. $\mathrm{B}$ domains unfold at forces larger than $2 \mathrm{nN}$ - a strength reminiscent of breaking a covalent bond. In comparison, pili domains of FimA from Actinomyces oris have been shown to unfold at $\sim 0.7 \mathrm{nN}^{23}$ and cohesin domains from cellulosomal bacteria unfold at $0.6 \mathrm{nN}^{24,25}$, both at similar force loading rates. Through sitedirected mutations, we demonstrate that this stability rests on the coordination of calcium $\left(\mathrm{Ca}^{2+}\right)$ ions. Each $\mathrm{B}$ domain coordinates three $\mathrm{Ca}^{2+}$ ions in different positions. When these are chelated from the domain their mechanostability drastically decreases by a factor of four - yet forces are still in the vicinity of $0.6-0.8 \mathrm{nN}$. Systematically incapacitating the $\mathrm{Ca}^{2+}$ coordination sites revealed which $\mathrm{Ca}^{2+}$ ion is most important to the mechanostability. Furthermore, there are subtle differences between B domains from related organisms - even the same gene - opening multiple scenarios for their role in pathogen adhesion.

\section{Results}

The SdrG B1 domain unfolds at forces over $2 \mathrm{nN}$. The B domains from staphylococcal adhesin SdrG, B1, and B2, act as a linker between the N-terminal A region, where domains N2 and $\mathrm{N} 3$ bind host targets with extremely high resilience to mechanical force (Fig. 1a), and the C-terminus, which is covalently anchored to the bacterial peptidoglycan via a sortase motif (Figs. 1b, c). Thus, the $\mathrm{B}$ domains are located between an extremely mechanostable non-covalent interaction and a covalent bond, motivating our investigation into their force resilience. Initial experiments probed the unfolding forces of the SdrG B1 domain using its wild type (WT), adjacent ultrastable protein handle, the $\mathrm{N} 2$ and N3 domain (Fig. 1d). SdrG N2N3 binding a 15 aminoacid peptide from the N-terminus of fibrinogen $B$ (Fgß) withstands $>2 \mathrm{nN}$ in force. Alternatively, we used the clumping factor B N2 and N3 domains (ClfB) from S. aureus as a handle, which binds a 12 amino-acid C-terminal peptide of dermokine (DK) and conveniently has no B domains, as well as a higher unbinding force than SdrG (Key plasmids for this study were deposited with Addgene and can be found in Table 1). The unfolding forces of SdrG B1 were consistently in the range of $2 \mathrm{nN}$ (Figs. 1e, f). Traces containing no unfolding events before the handle ruptured indicate that sometimes SdrG B1 domain stability even exceeded that of the N2N3 handle. The contour length increment of the unfolding event matched the expected length for an unfolded B1 domain (110 amino acids $\times 0.365 \mathrm{~nm}$ per residue $-4 \mathrm{~nm}$ folded protein $=36 \mathrm{~nm}$ ). Previous cell-based force spectroscopy work on SdrG had described an event preceding complex rupture at comparable forces and extension increments - yet not identified it as a B domain 7 . The SdrG B1 domain was located on the cantilever, whose apex (radius $\sim 10 \mathrm{~nm}$ ) can only present a limited number of molecules. Yet, its unfolding appeared in almost every trace for more than $12 \mathrm{~h}(N=3712$ events), thus we conclude that it refolds. A dynamic force spectrum was acquired for the high-force unfolding of B1 (Fig. 1f), which was described well by the Bell-Evans (BE) model. Curiously, a second unfolding population with an identical contour length increment unfolding $\sim 600 \mathrm{pN}$ also emerged (shown in Fig. 2a), hinting at a second, weaker unfolding pathway (excluded from Fig. 1f).

$\mathrm{Ca}^{2+}$-binding sites govern the mechanostability of SdrG B1. A crystal structure of the SdrG B1 domain is not available to date. Fortunately, a homolog's (SdrD from Staphylococcus aureus) B1 domain structure (PDB 4JDZ, alignment see Supplementary Fig. 1) had been determined previously ${ }^{26}$. Homology models of SdrG B1 and B2 could be constructed and were equilibrated using QwikMD configured NAMD molecular dynamics simulation ${ }^{27-29}$. Notably, the B domain adopts an Immunoglobulin (Ig)-like fold containing exclusively $ß$-strands (Fig. 1c). Furthermore, each B domains coordinates three calcium ions, which were numbered as displayed in Fig. 1b and Fig. 3a. Calcium one to three (Ca1-Ca3) are coordinated mostly via negatively charged side chains. Ca1 is enclosed in a loop, Ca2 lies more solvent exposed and closer to $\mathrm{Ca} 3$, which is coordinated by two aspartic acids on the $\mathrm{N}$ - and $\mathrm{C}$-terminal $\mathrm{B}$-strands that close the fold (see Figs. 3a, b).

The presence of calcium is relevant for both folding and stability of many protein domains ${ }^{30}$. Thus, to remove $\mathrm{Ca}^{2+}$ ions from the domain, buffers were exchanged introducing a high concentration of the chelating agent ethylenediaminetetraacetic acid (EDTA), which binds divalent ions. When probed in $10 \mathrm{mM}$ EDTA, the stability of SdrG B1 dramatically decreased, and the previously described weak unfolding event $\sim 600 \mathrm{pN}$ appeared exclusively (a set of representative force extension curves is shown in Supplementary Fig. 2, for contour length diagram alignments see Supplementary Fig. 3). The SdrG:Fgß interaction remained unaffected by EDTA, despite its $\mathrm{Ca}^{2+}$-binding loop. When using citric acid - a more physiological, but milder chelating agent instead of EDTA, the same weak state emerged (Supplementary Fig. 4), although even at $100 \mathrm{mM}$ citric acid $\sim 40 \%$ of domains still unfolded from the strong state. As the contour length increment of the weak state remained unchanged compared to the strong unfolding, the B1 domain was still folded in EDTA. The depletion of calcium switched it into a mechanically weaker state, yet it was unclear how many $\mathrm{Ca}^{2+}$ were chelated from $\mathrm{SdrG}$ B1. The strong state with unfolding events exclusively around $2 \mathrm{nN}$, was recovered after returning to $10 \mathrm{mM} \mathrm{Ca}^{2+}$ (example traces shown in Fig. 2a). This calcium induced stability switching could be repeated for multiple cycles, as shown in Fig. 2b. After EDTA chelation, applying high concentrations of $\mathrm{Mg}^{2+}$ did not change SdrG B1 weak state unfolding behavior. Even at $18 \mathrm{mM}$, 


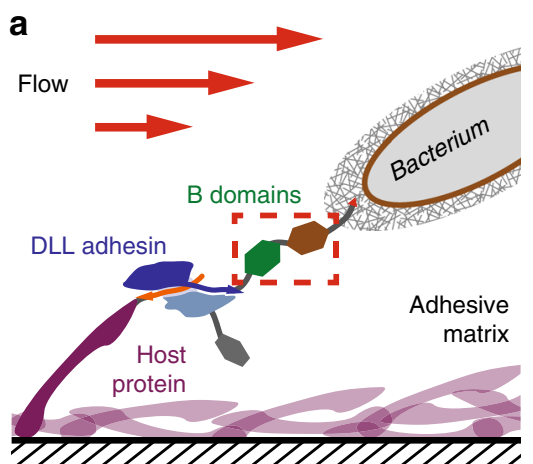

d

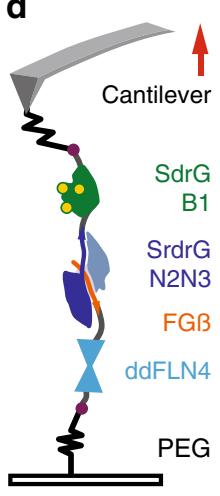

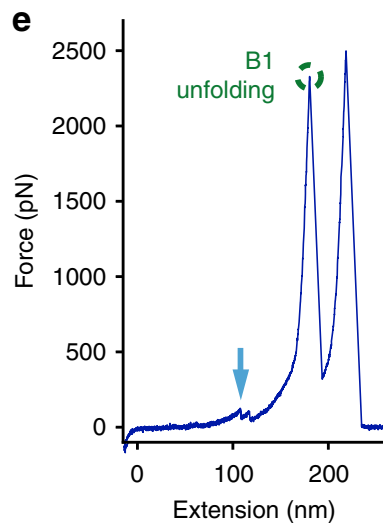

b

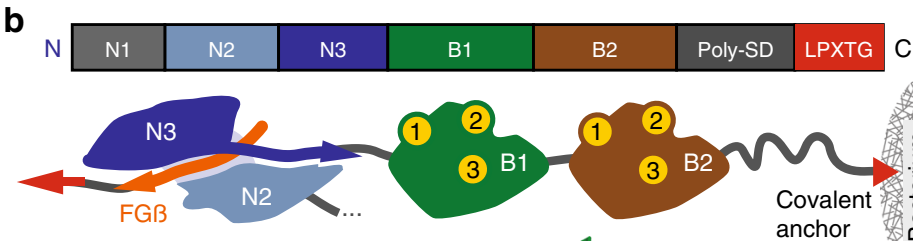

c
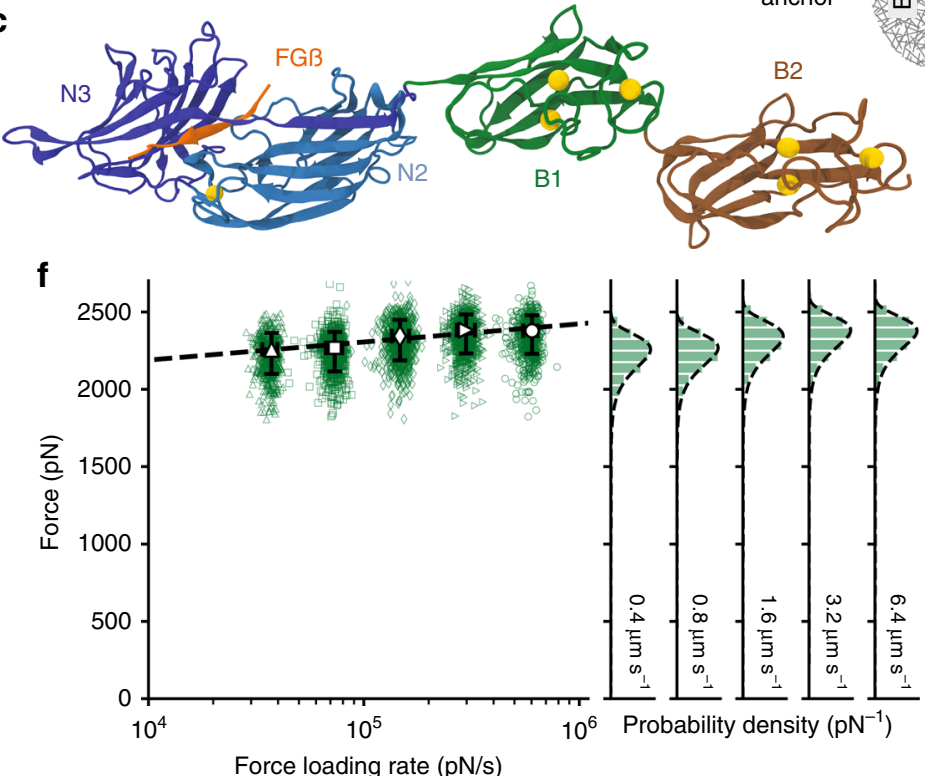

Fig. 1 Staphylococcal B domains are extremely resilient to mechanical force. a B domains, here from SdrG, are the link between the extremely strong interaction between the tip adhesin domain N2N3 (blues) binding a peptide (orange) presented by a host protein covering a surface (in this case fibrinogen, purple). b SdrG gene (top) and schematic (bottom): N-terminal N1 domain may be cleaved proteolytically, followed by the N2 and N3 domain that bind FgB, B1 (green), and B2 (brown) each coordinate three $\mathrm{Ca}^{2+}$ ions and connect to the SD repeat region that gives the adhesin its name. The adhesin is covalently anchored to the bacterium's peptidoglycan via a sortase motif (red). c MD equilibrated structure of the SdrG N2N3 domains connected to the fully B-sheet Ig-like folds of the B1 and B2 domain (modeled from the homolog SdrD B1), each B domain coordinates three Ca ${ }^{2+}$ ions (yellow). d AFM-SMFS assay: covalent surface anchoring through polyethylene glycol (PEG) via the ybbr-tag (purple) using Fgß (orange)-ddFLN4 (cyan)ybbr to probe SdrG N2N3-B1-ybbr on the cantilever. e Single force-extension trace at $0.8 \mu \mathrm{m} \mathrm{s}^{-1}$ with unfolding of the ddFLN4 fingerprint (cyan arrow) at $100 \mathrm{pN}$, followed by the SdrG B1 domain (green circle) at $>2 \mathrm{nN}$ (with the expected contour length increment of $\sim 36 \mathrm{~nm}$ ). Finally the SdrG N2N3:Fgß complex dissociates, allowing the cantilever to relax to zero force. $\mathbf{f}$ Dynamic force spectrum for the unfolding of the SdrG B1 domain at retraction velocities: $0.4 \mu \mathrm{m} \mathrm{s}^{-1}$ (triangles, $N=574$ ), $0.8 \mu \mathrm{m} \mathrm{s}^{-1}$ (squares, $N=742$ ), $1.6 \mu \mathrm{m} \mathrm{s}^{-1}$ (diamonds, $N=878$ ), $3.2 \mu \mathrm{m} \mathrm{s}^{-1}$ (forward triangles, $N=789$ ), $6.4 \mu \mathrm{m} \mathrm{s}^{-1}$ (circles, $N=729$ ). The high $\mathrm{N}$ value suggests that the B1 domain refolds on the cantilever. A Bell-Evans model fit (dashed line, $\Delta \mathrm{x}=0.082 \mathrm{~nm}$, $k_{\text {off }}{ }^{0}=3.8 \mathrm{E}-17 \mathrm{~s}^{-1}$ ) through the most probable rupture force and force loading rate per velocity (large open markers, errors given as full-width at half maximum for each distribution) confirms the expected log-linear behavior

\section{Table 1 Key plasmids with Addgene accession numbers}

\section{Plasmid}

pET28a-SdrG_N2N3-HIS-ybbr

pET28a-CIfB_N2N3-HIS-ybbr

pET28a-SdrG_N2N3-B1-B2-HIS-ybbr

pET28a-SdrG_N2N3-B1-HIS-ybbr

pET28a-MGGG-ybbr-HIS-SdrG_B1-DK

pET28a-MGGG-ybbr-HIS-SdrG_B2-DK

pET28a-MGGG-ybbr-HIS-SdrD_B1-DK

$\mathrm{Mg}^{2+}$ was unable to occupy the $\mathrm{Ca}^{2+}$ coordination sites (see Supplementary Fig. 5). The dynamic force spectra for both weak and strong states, shown in Fig. 2c, were determined with a single cantilever. Notably, the dependency of the most probable rupture force on the natural logarithm of the force loading rate in the $\mathrm{BE}$ model is almost parallel for both states (strong state: $\Delta \mathrm{x}=$ $0.083 \mathrm{~nm}, k_{\text {off }}^{0}=2.8 \mathrm{E}-17 \mathrm{~s}^{-1}$, weak state $\Delta \mathrm{x}=0.071 \mathrm{~nm}, k_{\text {off }}^{0}=$ $\left.0.011 \mathrm{~s}^{-1}\right)$, reflected in similar distances to the transition state $\Delta \mathrm{x}$ (within $\sim 17 \%$ of each other), whereas the large difference in unfolding force is given through the zero force off-rates $k_{\text {off }}^{0}$, which differ by $>14$ orders of magnitude.

After they had been exposed to EDTA, inducing the weak state, SdrG B1 domains were returned to $\mathrm{Ca}^{2+}$-free buffers $(25 \mathrm{mM}$ TRIS, $75 \mathrm{mM} \mathrm{NaCl}, \mathrm{pH} 7.4$ ) and found mostly in the strong state. This puzzling contradiction of $\mathrm{Ca}^{2+}$-dependent folding could be explained by trace amounts of contaminating $\mathrm{Ca}^{2+}$ in the buffer, estimated to be in the $\mathrm{nM}$ range (manufacturer's specifications, see Methods). Thus, the affinity of SdrG B1 for $\mathrm{Ca}^{2+}$ must be extremely high. Moving to higher purity $\mathrm{Ca}^{2+}$-free reagents (see Methods), a trace $\mathrm{Ca}^{2+}$ concentration low enough to keep the domains in the weak state was achieved. Previously, the $\mathrm{Ca}^{2}$ + -dependent folding and thus $\mathrm{Ca}^{2+}$ affinity of homologous $\mathrm{B}$ domains from $S$. aureus $\mathrm{SdrD}$ had been measured ${ }^{21,31}$. As our AFM experiments could clearly discern between the strong $\mathrm{Ca}^{2+}$ saturated and weak, $\mathrm{Ca}^{2+}$-depleted state, we titrated the amount of $\mathrm{Ca}^{2+}$ to estimate the affinity of the weak state SdrG B1 domain for $\mathrm{Ca}^{2+}$ by plotting the fraction of strong to weak state events against $\mathrm{Ca}^{2+}$ concentration. The inflection point was below $1 \mathrm{nM}$, within our conservative estimate of $\mathrm{Ca}^{2+}$ concentration uncertainty, hinting at a sub-nM $\mathrm{K}_{\mathrm{D}}$ for $\mathrm{Ca}^{2+}$ (Figs. 2d, e). 

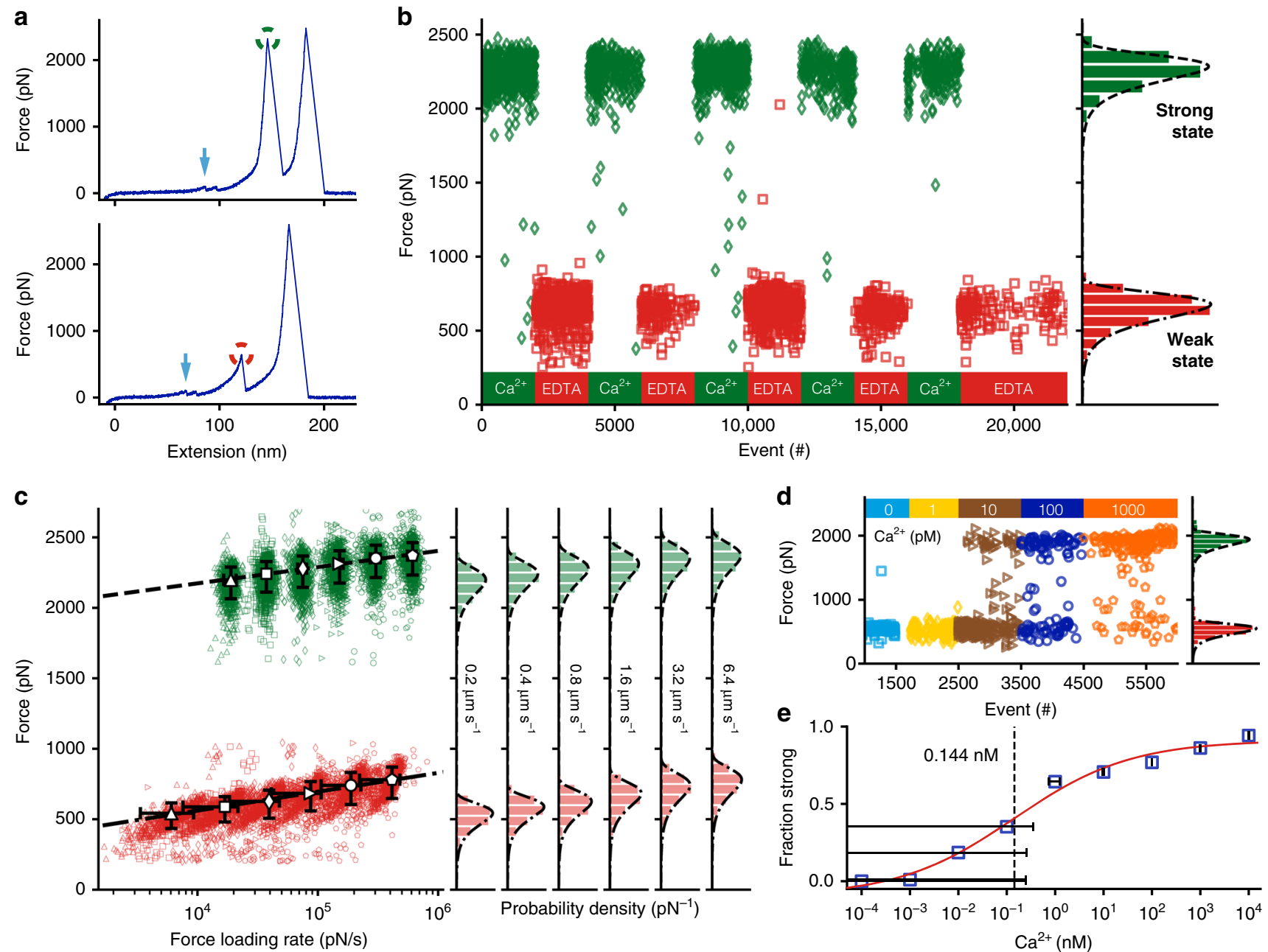

Fig. 2 B domain stability and unfolding force are governed by calcium. a SdrG B1 unfolds in $10 \mathrm{mM} \mathrm{Ca}{ }^{2+}$ at over $2 \mathrm{nN}$ (strong state, green circle) and here at $\sim 650 \mathrm{pN}$ in the presence of $10 \mathrm{mM}$ EDTA (weak state, red circle) at a retraction velocity of $1.6 \mu \mathrm{m} \mathrm{s}{ }^{-1}$, after ddFLN4 fingerprint unfolding (cyan arrows). $\mathbf{b}$ $\mathrm{SdrG}$ B1 domain stabilities can be cycled repeatedly by alternate application of $\mathrm{Ca}^{2+} 10 \mathrm{mM}$ (green diamonds) and EDTA $10 \mathrm{mM}$ (red squares). c Dynamic force spectrum of the weak and strong state stabilities. Strong state (green) in $10 \mathrm{mM} \mathrm{Ca}^{2+}: 0.2 \mu \mathrm{m} \mathrm{s}^{-1}$ (triangles, $N=848$ ), $0.4 \mu \mathrm{m} \mathrm{s} \mathrm{s}^{-1}$ (squares, $N=$ 1128), $0.8 \mu \mathrm{m} \mathrm{s}^{-1}$ (diamonds, $N=1162$ ), $1.6 \mu \mathrm{m} \mathrm{s}^{-1}$ (forward triangles, $N=1202$ ), $3.2 \mu \mathrm{m} \mathrm{s}^{-1}$ (circles, $N=1039$ ), $6.4 \mu \mathrm{m} \mathrm{s}^{-1}$ (pentagons, $N=1129$ ), $B E$ fit (dashed line, $\left.\Delta x=0.083 \mathrm{~nm}, k_{\text {off }}^{0}=2.8 \mathrm{E}-17 \mathrm{~s}^{-1}\right)$. Weak state (red) in $10 \mathrm{mM}$ EDTA (markers as before): $0.2 \mu \mathrm{m} \mathrm{s}^{-1}(N=664), 0.4 \mu \mathrm{m} \mathrm{s}-1(N=767)$, $0.8 \mu \mathrm{m} \mathrm{s}^{-1}(N=953), 1.6 \mu \mathrm{m} \mathrm{s}^{-1}(N=922), 3.2 \mu \mathrm{m} \mathrm{s}^{-1}(N=861), 6.4 \mu \mathrm{m} \mathrm{s}^{-1}(N=1007)$, BE fit (dashed-dotted line, $\left.\Delta x=0.071 \mathrm{~nm}, k_{\text {off }}{ }^{0}=0.011 \mathrm{~s}^{-1}\right) . \mathbf{d}$ $\mathrm{Ca}^{2+}$ titration experiment with SdrG B1 immobilized on a surface in varying $\mathrm{Ca}^{2+}$ concentrations, starting from EDTA $10 \mathrm{mM}^{2}$ to $\mathrm{Ca}^{2+}$-free buffer in which all B1 unfolding events show the weak state. At $10 \mathrm{pM} \mathrm{Ca}{ }^{2+}$ the strong state starts to appear constituting the majority of unfolding events at $1000 \mathrm{pM}$ $\mathrm{Ca}^{2+}$. There are almost no unfolding events in an intermediate regime $(N=995)$. e Affinity estimate of $\mathrm{SdrG} B 1$ from combined Ca ${ }^{2+}$ titration experiments, showing the fraction of all curves with $B$ domain unfolding events in the strong state. A four-parameter logistic regression fit (red line) yields an inflection point of $0.144 \mathrm{nM}$, pointing to a sub-nM K $\mathrm{D}_{\mathrm{D}}$ of $\mathrm{SdrG} \mathrm{B1}$ for $\mathrm{Ca}^{2+}$ in the weak state, albeit concentration uncertainties (error bars as trace $\mathrm{Ca}^{2+}$ uncertainty in buffer and $1 \%$ dilution error, $N=1703$ ) in the sub-nM range are very high

Incapacitating $\mathrm{Ca}^{2+}$-binding sites lowers SdrG B1 stability. Clearly, the addition of EDTA, i.e., the removal of $\mathrm{Ca}^{2+}$, weakened the B1 domain. However, we could not discern if all, or only a fraction of the three $\mathrm{Ca}^{2+}$ ions were removed. To determine how and which $\mathrm{Ca}^{2+}$ ions were crucial to the stability, mutants lacking the amino acids required to coordinate each $\mathrm{Ca}^{2}$ ${ }^{+}$were produced (hereafter $\mathrm{Ca} 1 \mathrm{KO}, \mathrm{Ca} 2 \mathrm{KO}, \mathrm{Ca} 3 \mathrm{KO}$, respectively), shown in Figs. 3a, b. To map the interplay between the loops, additionally all permutations of mutants with two $\mathrm{Ca}^{2+}$ sites deleted, leaving only a single $\mathrm{Ca}^{2+}$ bound, were created (Ca1,2KO; Ca2,3KO; Ca1,3KO, overview in Supplementary Fig. 1b). All mutants were probed in a single AFM-SMFS experiment using the same cantilever in both $10 \mathrm{mM}$ EDTA and $10 \mathrm{mM} \mathrm{Ca}^{2+}$, to compare absolute stabilities ${ }^{25,32}$.
Results are shown in Fig. 3c (detailed distributions in Supplementary Fig. 6): Ca1 is coordinated by the largest number of negatively charged amino acids side chains (Fig. 3a), which intuitively would make it the most important, and a likely candidate to stay bound in chelating conditions. Interestingly, the Ca1KO mutant was only half as strong compared with the WT in $\mathrm{Ca}^{2+}$. Ca2KO was only $\sim 10 \%$ weaker than the WT in $\mathrm{Ca}^{2+}$. For both $\mathrm{Ca} 1 \mathrm{KO}, \mathrm{Ca} 2 \mathrm{KO}$ the weak state in EDTA remained at WT strength. Ca3KO showed the most drastic change both in $\mathrm{Ca}^{2+}$ and EDTA, as unfolding forces dropped by an order of magnitude. When incapacitating two $\mathrm{Ca}^{2+}$-binding sites at a time (Fig. 3d) Ca1,2KO behaved similar to the Ca1KO mutant, hinting that $\mathrm{Ca} 2$ could still be occupied by a $\mathrm{Ca}^{2+}$ ion as it interacts with parts of the peptide backbone and an aspartic acid 

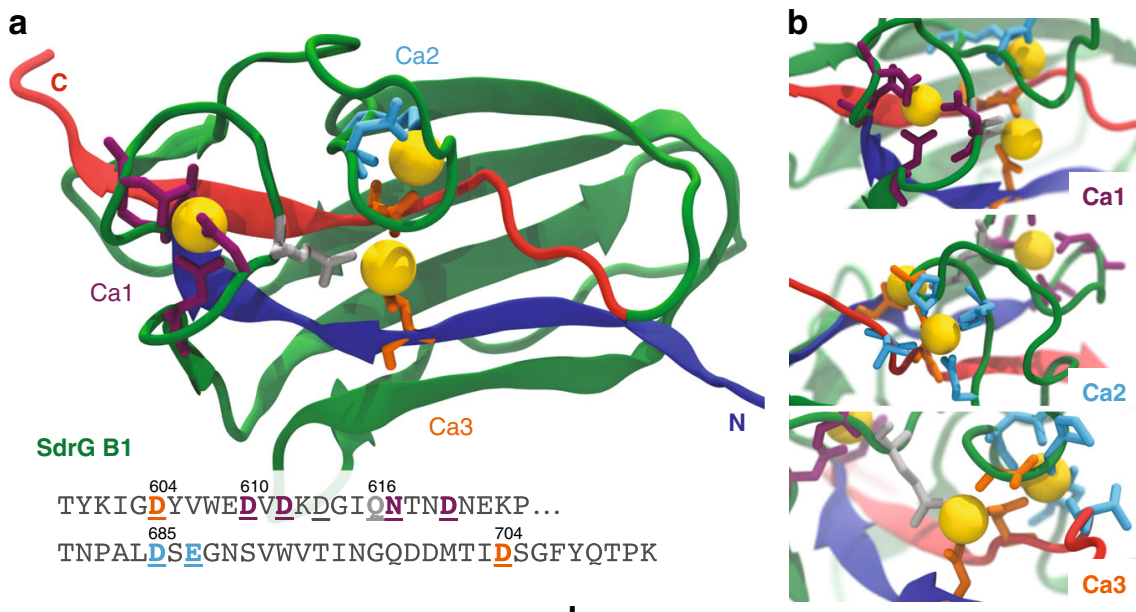

C

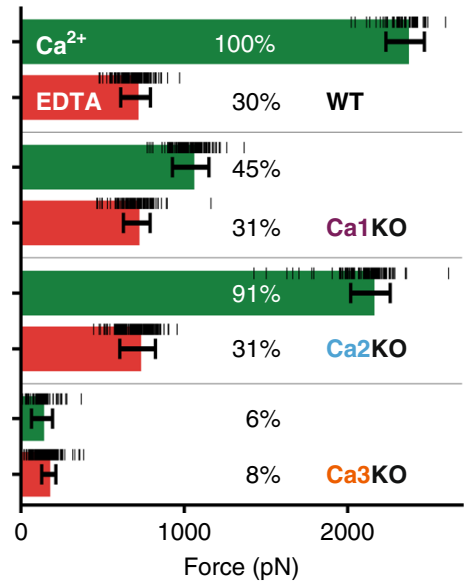

d

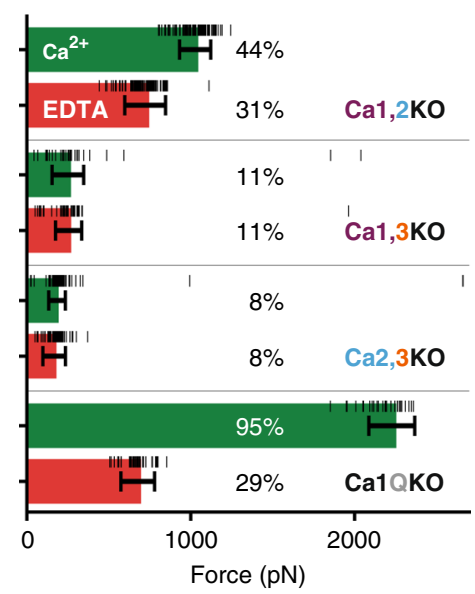

Fig. $3 \mathrm{Ca}^{2+}$ loop three is most important to SdrG B1 domain stability. a Equilibrated homology model of SdrG B1, with N-and C-terminal B-strands marked in blue and red, respectively. $\mathrm{Ca}^{2+}$ coordinating amino-acid side chains shown as sticks. $\mathrm{Ca}^{2+}$ binding sites one to three are marked (Ca1 purple, $\mathrm{Ca} 2 \mathrm{cyan}$, $\mathrm{Ca} 3$ orange). A conserved glutamine bridge (light gray) connects the $\mathrm{Ca}$ l loop to $\mathrm{Ca}$. Amino acids mutated to remove respective loops are underlined in the sequence shown. $\mathbf{b}$ Closeup of each $\mathrm{Ca}^{2+}$ binding loop, including non-sidechain coordinating residues in stick representation. $\mathbf{c}$, $\mathbf{d}$ Comparison of absolute unfolding forces of mutated SdrG B1 in $10 \mathrm{mM}$ EDTA (red) and $10 \mathrm{mM} \mathrm{Ca}^{2+}$ (green) with a single cantilever, also given in percentage of the WT strong state. Errors are the full-width at half maximum of the BE fits for each unfolding force distribution (see Supplementary Fig. 6), underlying raw force datapoints are shown as black horizontal lines. The single loop knockouts in (c) show that Ca3 is crucial for overall stability and most likely remains bound in the weak state. Once Ca3 is removed, weak state forces drop to only 6-8\% of WT strength. Removing Ca1 or Ca2 or both (d) supports this as the weak state remains at $31 \%$ of WT. The glutamine bridge (Ca1QKO) seems to be of minor importance for the overall domain stability

of Ca3. These contacts might be sufficient for coordination (see detailed coordination sites in Supplementary Fig. 7). Ca1,3KO and $\mathrm{Ca} 2,3 \mathrm{KO}$ were drastically weaker than the WT, comparable to $\mathrm{Ca} 3 \mathrm{KO}$. The removal of a conserved glutamine bridge (Ca1QKO) between $\mathrm{Ca} 1$ and $\mathrm{Ca} 3$ only led to a minor decrease in domain strength.

In summary, $\mathrm{Ca} 3$ is most crucial for overall B domain stability, essential to establishing the $600 \mathrm{pN}$ weak state, and most likely stays bound in the presence of EDTA. Adding Ca2 increases the stability to over $1 \mathrm{nN}$, whereas adding Cal boosts it to over $2 \mathrm{nN}$. Even in very dilute $\mathrm{Ca}^{2+}$, well below the $\mathrm{K}_{\mathrm{D}}$ in the titration series, we never observed more than a handful of events in a force range comparable to Ca1KO and Ca2KO (see Fig. 2d). Instead, SdrG B1 immediately occupied the strongest state. Thus, we propose that binding of $\mathrm{Ca} 1$ and $\mathrm{Ca} 2$ must be highly cooperative.

Homologous B domains show similar unfolding forces. SdrG contains a second B domain (B2), whose sequence is $45 \%$ identical to B1 (alignment, see Supplementary Fig. 1). An equilibrated homology model is shown in Fig. 4a. The crystalized SdrD B1 domain, shown equilibrated in Fig. 4b, was investigated, too.
When measured in $10 \mathrm{mM} \mathrm{Ca}^{2+}$, SdrG B2 and SdrD B1 showed a similar, $2 \mathrm{nN}$ stability. However, in EDTA a weak unfolding event with their expected contour length appeared only rarely. Most curves contained no discernible unfolding peak (above our detection limit around $20 \mathrm{pN}$ ), hinting at a complete unfolding of the domains, a marked difference from to the mere weakening of SdrG B1. The $\mathrm{Ca}^{2+}$-EDTA switching, for SdrD B1 (Fig. 4c) thus resulted in very few weak events detected in EDTA, which showed a bimodal unfolding force distribution that was described well by a superposition of two BE fits (Eq. 1, Fig. 4c).

Given their highly similar structure, this result was unexpected. Subtle differences in B domains must give them diverging properties. When comparing all three domains, further differences emerged: the $\mathrm{Ca}^{2+}$ affinity of SdrD B1 was slightly lower than for SdrG B1 and lowest for SdrG B2 (fraction of all curves with folded domain, i.e., strong and weak in low $\mathrm{Ca}^{2+}$ : SdrG B1 $87 \%$, SdrD B1 80\%, SdrG B2 33\%, Fig. 4d). Intriguingly, SdrG $\mathrm{B} 1$ and adjacent B2 have clearly separated regimes at which they switch to their strongest state. A comparison of the absolute mechanostabilities of SdrG B1, SdrG B2, and SdrD B1 was conducted with a single cantilever ${ }^{25}$, using ClfB as a handle. The results are depicted in Fig. 4e. SdrD B1 and SdrG B2 exhibit 

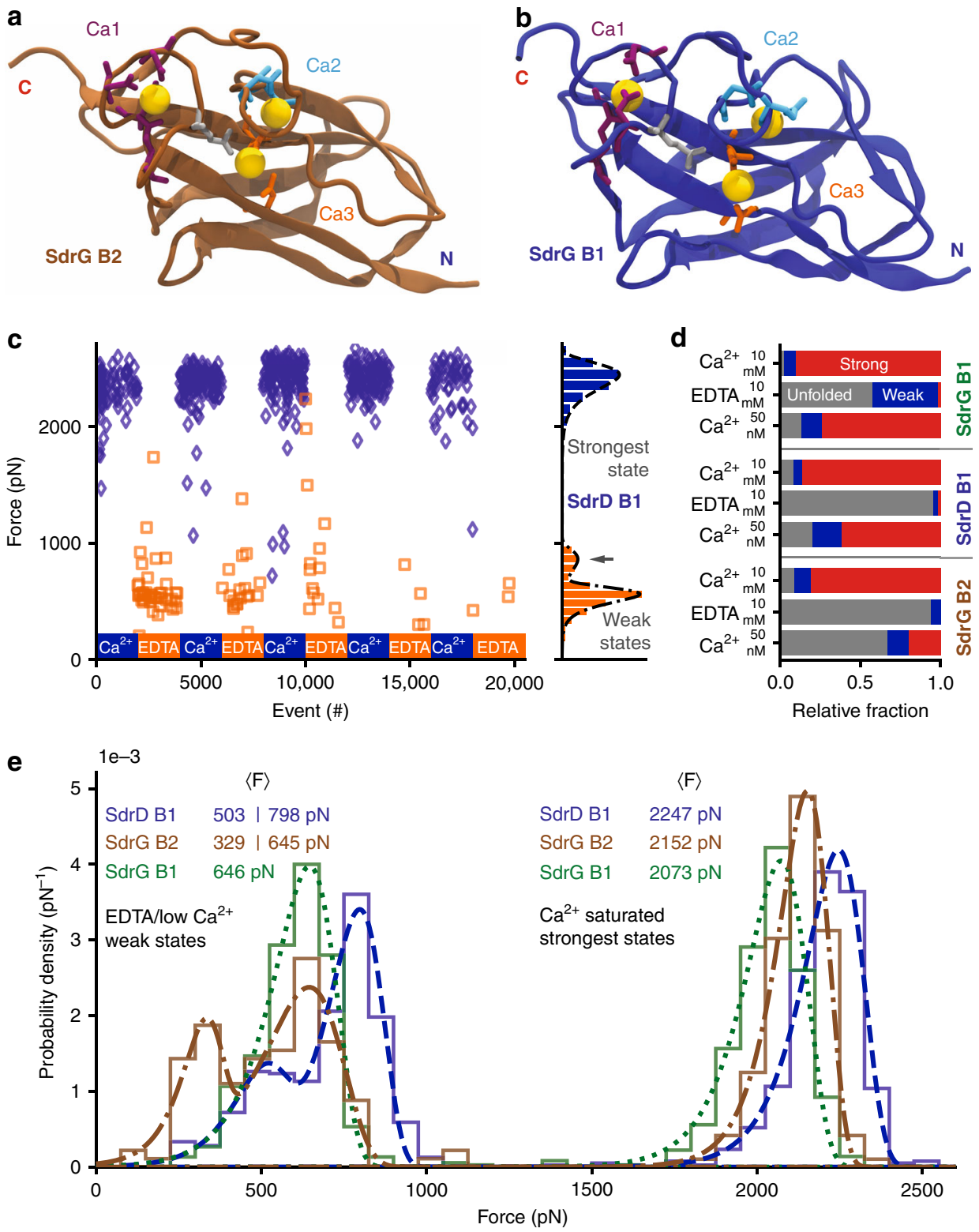

Fig. 4 SdrG B2 and SdrD B1 are even stronger than SdrG B1 and have different Ca ${ }^{2+}$ affinities. a Equilibrated homology model of SdrG B2 (brown) with $\mathrm{Ca}^{2+}$ (yellow) binding loops one to three marked, coordinating amino-acid side chains shown as sticks (coloring as in Fig. 3a). b Equilibrated crystal structure of SdrD B1 (blue, PDB 4JDZ). c Cycling of SdrD B1 between $10 \mathrm{mM} \mathrm{Ca}^{2+}$ and $10 \mathrm{mM}$ EDTA, a strong state in $\mathrm{Ca}^{2+}$ over $2000 \mathrm{pN}$ emerges. The weak state is bimodal peaking at $\sim 550 \mathrm{pN}$ and $\sim 850 \mathrm{pN}$ (gray arrow), fit with a superposition of two BE p(F) functions (see methods). Very few unfolding events appear in $10 \mathrm{mM}$ EDTA, thus the $\mathrm{Ca}^{2+}$-chelation must be sufficient to completely unfold most SdrD B1 domains. The strong state is also bimodal in low $\mathrm{Ca}^{2+}$ conditions see Supplementary Fig. 8. d Relative fraction of states in different buffers: $10 \mathrm{mM} \mathrm{Ca}{ }^{2+}, 10 \mathrm{mM}$ EDTA, and $50 \mathrm{nM} \mathrm{Ca}{ }^{2+}$ (applied after $10 \mathrm{mM}$ EDTA) for SdrG B1, B2 and SdrD B1 compared with a single cantilever: no unfolding event (unfolded protein, gray), weak state (<1000 pN, blue), strong state $\left(>1500 \mathrm{pN}\right.$, red). Almost all SdrD B1 and SdrG B2 domains are unfolded in EDTA and only a fraction refolds in low Ca ${ }^{2+}$. SdrG B2 has the lowest affinity for $\mathrm{Ca}^{2+}$ as it shows the least folded events in low $\mathrm{Ca}^{2+}$. e Comparison of relative unfolding forces of all $\mathrm{B}$ domains at $1.6 \mu \mathrm{m} \mathrm{s}^{-1}$ with a single cantilever. The strongest state of SdrD B1 (blue, dashed line) is the most mechanostable, followed by SdrG B2 (brown, dash-dotted line), then SdrG B1 (green, dotted line). The weak states of SdrD B1 and SdrG B2 are bimodal and best described with a superposition of two BE fits, whereas SdrG B1 only has a single weak state

bimodal unfolding force distributions in the weak state. The strong states of these domains are also bimodal in low $(\sim 50 \mathrm{nM})$ $\mathrm{Ca}^{2+}$ concentrations (not shown here, see Supplementary Fig. 8), hinting at a separable, stepwise binding of $\mathrm{Ca}^{2+}$. In saturating $10 \mathrm{mM} \mathrm{Ca}{ }^{2+}$ conditions, all domains have a unimodal unfolding force distribution, with $\mathrm{SdrD}$ B1 being the strongest, followed by $4 \%$ weaker SdrG B2, and 8\% weaker SdrG B1. Although SdrG B2 has a lower affinity for $\mathrm{Ca}^{2+}$ than SdrG B1 it is clearly stronger, demonstrating that $\mathrm{B}$ domain $\mathrm{Ca}^{2+}$ affinity is not correlated with its mechanostability.

\section{Discussion}

Why have B domains evolved these exceptional mechanical stabilities? Their extreme mechanostability may be rationalized in context of the ligand-binding region, which they connect to the bacterium. In the case of SdrG, the mechanical stability of the 
interaction between the $\mathrm{N} 2$ and $\mathrm{N} 3$ domains binding their Fgß target is independent of the B domains, as they can be deleted from the construct without lowering the interaction rupture force ${ }^{9}$. Contrary to DLL adhesins, recently studied thioester domain (TED) adhesins attach to their human targets through a covalent isopeptide bond ${ }^{33-35}$. Covalent bonds are mechanically stronger and irreversible compared with the non-covalent, spontaneously reversible, DLL attachment $\left(\mathrm{K}_{\mathrm{D}}\right.$ for SdrG N2N3: Fgß $\sim 400 \mathrm{nM})^{5}$. Isopeptide bonds are covalent amide bonds between amino-acid side chains that stabilize a fold, or connect two proteins. TEDs and collagen-binding MSCRAMMs are linked by Ig-like and $\mathrm{Ca}^{2+}$ coordinating folds such the Spy0128 pilus of $S$. pyogenes, which contain intramolecular isopeptide bonds $s^{36,37}$. These block the mechanical extension of Spy0128 ${ }^{38}$. Notably, another Ig-like domain from S. pyogenens fibronectin binding protein (fba2) follows a remarkably similar fold to the $\mathrm{B}$ domains here (structural alignment in Supplementary Fig. 9). Their most striking difference is that fba2 is stabilized by the isopeptide bond locking the $\mathrm{N}$ and $\mathrm{C}$-terminal $\mathrm{B}$-sheet together. The SpyTag/Catcher covalent labeling system was derived from this system ${ }^{39}$. In SdrG B1 and its homologs, the coordinated $\mathrm{Ca}^{2+}$ ions are covering these strands and give the domain extraordinary mechanostability. The rigidity of $\mathrm{B}$ regions, especially those containing isopeptide bonds, such as the collagen adhesin Cna of $S$. aureus ${ }^{40}$, has been proposed to project the ligand-binding region away from the bacterial surface toward the hos $\mathrm{t}^{37,41,42}$, which could also be a function of the $\mathrm{B}$ domains investigated here. Isopeptides, as covalent bonds, are stronger than the B domain fold, making them completely resistant to unfolding, ultimately resulting in a stiff, rigid stalk. In contrast, B domains can un- and refold. Both systems achieve high mechanical stability, however, they differ in what is best addressed as malleability. SdrG B domains can act as a "mechanical shock dissipater" under tension, as previously proposed for pili domains by Echelman et al. ${ }^{23}$. Domains unfold to buffer transient stress, e.g., caused by shear flow, on the ligand-binding region at the tip and regenerate when tension recedes.

At physiological $\mathrm{Ca}^{2+}$ concentrations (free $\mathrm{Ca}^{2+}$ ions in human blood on the order of $1 \mathrm{mM}^{43}$ ) at least SdrG B1 would be found almost exclusively in its strong state. However, the mechanical stabilities of the strongest state of SdrG B1 and B2 are on the order of the interaction of the SdrG N2N3 domains with fibrinogen. Sometimes the interaction is not sufficient to unfold the $\mathrm{B}$ domain at force loading rates around $10^{4} \mathrm{pN} \mathrm{s}^{-1}$ - in clear conflict with the proposed shock dissipater by unfolding hypothesis. The BE fits (see Supplementary Fig. 10) show that the SdrG B1 domain's unfolding force dependency on the force loading rate has a less steep slope than the SdrG adhesin. Extrapolating from this range, the $\mathrm{B}$ domains would reliably be weaker than the N2N3 receptor-ligand interaction at higher force loading rates exceeding $10^{6} \mathrm{pN} \mathrm{s}^{-1}$. In this range, at least SdrG B1 could fulfill a shock dissipater function. One could speculate that SdrG B1 only unfolds when rapid changes in load are stressing the SdrG adhesin, while letting slow changes in force act on the tip adhesin, thus acting as a low-pass filter for stress: a strongand-sudden load dissipater.

Many MSCRAMM adhesins contain more than one B domain (e.g., SdrD has five B domains in total). Previous work suggested that these have different individual $\mathrm{Ca}^{2+}$ affinities ${ }^{21,31}$. In the case of SdrG B1 and B2 have comparable unfolding forces, yet different $\mathrm{Ca}^{2+}$ affinities, despite $45 \%$ sequence identity and high similarity in the $\mathrm{Ca}^{2+}$ binding sites. Different $\mathrm{B}$ domains in the same adhesin thus may have specifically tuned functions. The varied $\mathrm{Ca}^{2+}$ affinities may control their mechanical strength, ensuring that one domain is preferentially in the weak, while another one occupies the strong or an intermediate state, as in the case of SdrD B1 and SdrG B2, whose intermediate state strengths would be ideal to dissipate stress.

The mechanism that governs these extreme mechanical unfolding forces is clearly dependent on the presence of $\mathrm{Ca}^{2+}$. The coordination of $\mathrm{Ca} 3$, connecting the parallel very $\mathrm{N}$ and $\mathrm{C}$ terminal, closing B-strands (see Fig. $3 \mathrm{a}$ in blue and red), is most crucial to overall B domain mechanostability. The molecular mechanism governing the comparable unbinding forces of the tip adhesin of SdrG N2N3:Fgß relies on the confined alignment of the backbone hydrogens bonds ( $\mathrm{H}$ bonds) between the target peptide and the enclosing locking strand in a shear geometry9. Analogously, one could propose that such an $\mathrm{H}$ bond-based mechanism stabilizes $\mathrm{B}$ domains. Indeed, the equilibration simulations show only few $\mathrm{H}$ bond contacts between the $\mathrm{N}$ and C-terminal $B$ strands, with most of them at the very C-terminus, below the Cal loop site. This geometry may change upon force application, but the mechanism that gives $\mathrm{B}$ domains their exceptional mechanostability most likely differs from SdrG:Fgß, in that the $\mathrm{Ca}^{2+}$ electrostatically protects the $\mathrm{H}$ bonds from breaking and locks them in a shear geometry. Alternatively, the coordination of $\mathrm{Ca}^{2+}$ ions may serve as a network though which forces propagate, diverting the load from the closing $\mathrm{N}$ - and Cterminal $\beta$-sheets.

$B$ domains are the mechanically strongest proteins examined to date, surpassing the stability of previously probed folds by at least a factor of two. B domains draw their stability from the coordination of $\mathrm{Ca}^{2+}$ ions, which are in some cases required for their refolding process. $\mathrm{B}$ domain $\mathrm{Ca}^{2+}$-dependent force resilience offers a blueprint to design extremely stable biomaterials with adjustable force response. In particular, SdrD B1 and SdrG B2 domain folding is tunable: from completely unfolded in EDTA, through a weak state in low $\mathrm{Ca}^{2+}$ unfolding around $500-800 \mathrm{pN}$, to over $2 \mathrm{nN}$ strong in high $\mathrm{Ca}^{2+}$. Each state can be induced through a $\mathrm{Ca}^{2+} /$ EDTA stimulus, respectively. Such properties may be fundamentally interesting as protein folding models that do not require aggressive denaturants to unfold, and more practically useful, e.g., in a stimuli-responsive protein hydrogel $^{44,45}$. A network of $B$ domains could withstand extreme forces when contracted and folded in $\mathrm{Ca}^{2+}$ but change into a flexible, extended polypeptide mesh when exposed to a $\mathrm{Ca}^{2+}$-chelating agent. Furthermore, SdrG B2 and SdrD B1 may be used for $\mathrm{Ca}^{2+}$ sensing, as their folding upon $\mathrm{Ca}^{2+}$ binding could be read out by monitoring FRET of dyes attached at their $\mathrm{N}$ - and C-termini.

Finally, the role of $\mathrm{B}$ domains in pathogen adhesion remains debatable. Roles such as extendable springs that stretch and contract, or a shock dissipater have been suggested ${ }^{41,46}$. However, the high SdrG B domain unfolding forces overlap with their respective receptor ligand unbinding at physiological $\mathrm{Ca}^{2+}$ concentrations and force loading rates around $10^{4} \mathrm{pN} \mathrm{s}^{-1}$, which prevent them from being reliable load dissipaters in this range. The weak state is ideally suited for this task, and at higher force loading rates, so are the strong states. It remains to be examined how $\mathrm{B}$ domains interact with each other ${ }^{46}$, respond to constant forces or low force loading rates, as well as changes in $\mathrm{pH}$, temperature or ionic strength, and what force the $\mathrm{B}$ domains exert when folding in the presence of $\mathrm{Ca}^{2+}$. The calciumdependent, ultrahigh mechanical stability of the B domain fold demonstrates to which extreme physical regimes pathogens evolved to invade their hosts.

\section{Methods}

Chemicals. All chemicals used were supplied by Carl Roth (Karlsruhe, Germany) or Sigma-Aldrich (St. Louis, MO, USA) if not specified explicitly.

Gene construction. The Dictyostelium discoideum $4^{\text {th }}$ filamin fingerprint domain (ddFLN4, UniProt: P13466, residues 549-649, with the internal cysteine mutated to 
serine), the Staphylococcus epidermidis SdrG N2N3, B1, and B2 domain genes (UniProt: Q9KI13), the Staphylococcus aureus ClfB N2 and N3 domains (UniProt: Q7A382); the SdrD B1 domain (from PDB 4JDZ with incomplete sequence, complete sequence in GenBank: WP_000934487 or obsolete UniProt entry: E5QTK7) were synthesized codon-optimized for expression in Escherichia Coli as linear DNA fragments (GeneArt - ThermoFisher Scientific, Regensburg, Germany) including suitable overhangs for Gibson assembly. Genes were inserted into pET28a Vectors with a hexahistidine-, ybbr-tag and in some cases a sortase motif via Gibson assembly ${ }^{47}$ (New England Biolabs, MA, USA). All point mutations, deletions, or additions of amino acids in all systems were created through polymerase chain reactions (Phusion Polymerase, New England Biolabs, MA, USA) with appropriate primers and finally blunt end ligation cloning using T4 Ligase (Thermo Scientific, MA, USA). Resulting open reading frames of all constructs were verified by DNA sequencing (Eurofins Genomics, Ebersberg, Germany). All protein sequences of constructs used in this study are listed in the Supplementary Information.

Important plasmids were deposited with Addgene (www.addgene.org) and are available through the following AddgeneIDs:

Protein expression and purification. All proteins were expressed in E. Coli NiCo21(DE3) (New England Biolabs, MA, USA). Bacterial starter cultures of $5 \mathrm{~mL}$ Lysogeny broth (LB) medium containing $50 \mu \mathrm{g} \mathrm{mL}^{-1}$ Kanamycin, were inoculated and grown overnight at $37^{\circ} \mathrm{C}$. These were added into in $100-200 \mathrm{~mL}$ of ZYM-5052 autoinduction media ${ }^{48}$ containing $100 \mu \mathrm{g} \mathrm{mL}{ }^{-1}$ Kanamycin and grown for $6 \mathrm{~h}$ at $37^{\circ} \mathrm{C}$ and cooled down, continuing overnight at $18^{\circ} \mathrm{C}$. For small-scale protein production, $8 \mathrm{~mL}$ cultures in ZYM-5052 autoinduction media were grown at $37^{\circ} \mathrm{C}$ overnight. Bacteria were harvested by centrifugation at $8000 \mathrm{~g}$, the supernatant was discarded, pellets were stored at $-80^{\circ} \mathrm{C}$ until purification.

All purification steps were performed at $4-8{ }^{\circ} \mathrm{C}$. The bacterial pellet was resuspended in lysis buffer ( $50 \mathrm{mM}$ TRIS, $50 \mathrm{mM} \mathrm{NaCl}, 5 \mathrm{mM} \mathrm{MgCl}_{2}, 0.1 \%(\mathrm{v} / \mathrm{v})$ Tween-20, 10\% (v/v) glycerol, $\mathrm{pH}$ 8.0) with $100 \mu \mathrm{gL}^{-1}$ lysozyme (Carl Roth, Karlsruhe, Germany). Cells were lysed by sonication (Sonoplus GM 70, with a microtip MS 73, Bandelin, Berlin, Germany). Insoluble parts were separated by centrifugation at $>40,000 \mathrm{~g}$ for at least $30 \mathrm{~min}$. The supernatant was sterile filtered $(0.45 \mu \mathrm{m}$, then $0.22 \mu \mathrm{m}$ pore size), adjusted to contain $20 \mathrm{mM}$ imidazole, and then loaded onto a Ni-NTA column (HisTrap FF $5 \mathrm{~mL}$ on a Äkta Start system, both GE Healthcare, MA, USA) for HIS-Tag purification and washed extensively $(25 \mathrm{mM}$ TRIS, $500 \mathrm{mM} \mathrm{NaCl}, 20 \mathrm{mM}$ imidazole, $0.25 \%$ (v/v) Tween-20, $10 \%$ (v/v) glycerol, $\mathrm{pH}$ 7.4). The protein was eluted in the same buffer, only different in containing $200 \mathrm{mM}$ imidazole and being at $\mathrm{pH}$ 7.8. Protein containing fractions were concentrated in centrifugal filters (Amicon, Merck, Darmstadt, Germany), exchanged into measurement buffer (TBS: $25 \mathrm{mM}$ Tris, $75 \mathrm{mM} \mathrm{NaCl}, \mathrm{pH}$ 7.4) by desalting columns (Zeba, Thermo Scientific, MA, USA), adjusted to $10 \%(\mathrm{v} / \mathrm{v})$ glycerol, and frozen in aliquots in liquid nitrogen to be stored at $-80{ }^{\circ} \mathrm{C}$ until thawed for experiments. Protein concentrations were determined by spectrophotometry at $280 \mathrm{~nm}$ with typical final concentrations of $30-1000 \mu \mathrm{M}$ (NanoDrop 1000, Thermo Scientific, MA, USA).

AFM sample preparation. More detailed AFM-SMFS protocol have been published previously ${ }^{16,49}$. In brief, AFM Cantilevers (Biolever Mini AC40TS, Olympus, Tokyo, Japan) and $24 \mathrm{mM}$ diameter cover glass surfaces (Menzel Gläser, Braunschweig, Germany) were modified with Aminosilane.

Glass surfaces: Glass surfaces were cleaned by sonication in $50 \%(\mathrm{v} / \mathrm{v}) 2$ propanol in ultrapure $\mathrm{H}_{2} \mathrm{O}$ for $10 \mathrm{~min}$, rinsed with ultrapure $\mathrm{H}_{2} \mathrm{O}$, and further cleaned and oxidized in $50 \%(\mathrm{v} / \mathrm{v}) \mathrm{H}_{2} \mathrm{O}_{2}$ and $50 \%(\mathrm{v} / \mathrm{v})$ of $30 \%(\mathrm{v} / \mathrm{v})$ sulfuric acid for $20 \mathrm{~min}$. Surfaces were washed in ultrapure $\mathrm{H}_{2} \mathrm{O}$, then ethanol. Surfaces were silanized by soaking in a solution of: $2 \%(\mathrm{v} / \mathrm{v})$ (3-aminopropyl)

dimethylethoxysilane (ABCR, Karlsruhe, Germany), $88 \%$ (v/v) ethanol, and $10 \%$ (v/v) ultrapure $\mathrm{H}_{2} \mathrm{O}$ under gentle shaking for $1 \mathrm{~h}$. Again, followed by two rinsing steps in ethanol, then rinsed in ultrapure $\mathrm{H}_{2} \mathrm{O}$, and afterwards dried in a gentle stream of nitrogen. Finally, surfaces were baked at $80^{\circ} \mathrm{C}$ for $45 \mathrm{~min}$. Glass surfaces were stored under Argon and typically used within 1 month.

Cantilevers: Following 15 min of UV-Ozone cleaning (UVOH 150 LAB, FHR Anlagenbau GmbH, Ottendorf-Okrilla, Germany), cantilevers were silanized, submerged in $1 \mathrm{~mL}$ (3-aminopropyl)-dimethylethoxysilane (APDMES, abcr, Karlsruhe, Germany) mixed with $1 \mathrm{~mL}$ ethanol and $5 \mu \mathrm{L}$ ultrapure $\mathrm{H}_{2} 0$ for 5 min. Each cantilever was rinsed in ethanol and subsequently in ultrapure $\mathrm{H}_{2} 0$. Finally, cantilevers were baked at $80^{\circ} \mathrm{C}$ for $1 \mathrm{~h}$ to be stored overnight under Argon and used in the following steps the next day.

Both glass surfaces and cantilevers were covered with $5 \mathrm{kDa}$ heterobifunctional a-Maleinimidohexanoic-PEG-NHS (Rapp Polymere, Tübingen, Germany) dissolved in $50 \mathrm{mM}$ HEPES (pH 7.5) at $25 \mathrm{mM}\left(125 \mathrm{mg} \mathrm{mL}^{-1}\right)$ for $30 \mathrm{~min}$. After rinsing surfaces and cantilevers in ultrapure water, $1 \mathrm{mM}$ coenzyme A (in $50 \mathrm{mM}$ sodium phosphate $\mathrm{pH} 7.2,50 \mathrm{mM} \mathrm{NaCl}, 10 \mathrm{mM}$ EDTA buffer) was applied to both for at least $1 \mathrm{~h}$. CoA functionalized surfaces and cantilevers stored in coupling buffer (50 mM sodium phosphate $\mathrm{pH} 7.2,50 \mathrm{mM} \mathrm{NaCl}, 10 \mathrm{mM}$ EDTA buffer) at $4^{\circ} \mathrm{C}$ were stable for $>4$ weeks.

When different protein constructs were compared with a single cantilever, up to 10 spatially separated spots were created using a silicone mask (CultureWell reusable gaskets, Grace Bio-Labs, Bend, OR, USA), cleaned by sonication in isopropanol and ultrapure $\mathrm{H}_{2} 0$, dried in a gentle stream of nitrogen, heated to $60^{\circ} \mathrm{C}$ and securely pressed onto a silanized microscopy slide $(76 \times 26 \mathrm{mM}$, Carl Roth, Karlsruhe Germany). Pegylation and CoA coupling in individual wells was achieved following identically to the protocol described above ${ }^{25}$.

These steps yielded cantilevers and surfaces covalently coated in PEG-CoA. Cantilevers and surfaces were rinsed in ultrapure water. Protein functionalization was achieved by covalently pulling down proteins via their ybbr-tag to CoA by the SFP enzyme coupling. The proteins of interest were diluted into TBS150 $(25 \mathrm{mM}$ Tris, $150 \mathrm{mM} \mathrm{NaCl}$, pH 7.4) supplemented with $10 \mathrm{mM} \mathrm{MgCl}_{2}$. Cantilevers were typically incubated with $50 \mu \mathrm{M}$ of protein of interest and $3 \mu \mathrm{M}$ Sfp phosphopantetheinyl transferase (SFP) for at least $1 \mathrm{~h}$. The glass surfaces were incubated with $5-15 \mu \mathrm{M}$ of protein construct of interest and 1-2 $\mu \mathrm{M}$ SFP for 30-60 min, depending on the desired surface density. Both samples were rinsed extensively with at least $60 \mathrm{~mL}$ measurement buffer (TBS75: $25 \mathrm{mM}$ Tris, $75 \mathrm{mM}$ $\mathrm{NaCl}, \mathrm{pH}$ 7.4) buffer before experiments.

AFM-SMFS. AFM-SMFS data were acquired on a custom-built AFM operated in closed loop by a MFP3D controller (Asylum Research, Santa Barbara, CA, USA) programmed in Igor Pro 6 (Wavemetrics, OR, USA). Experiments were conducted at room temperature (approximately $\left.25^{\circ} \mathrm{C}\right)$. Cantilevers were briefly $(<200 \mathrm{~ms})$ and gently $(<200 \mathrm{pN})$ brought in contact with the functionalized surface and then retracted at constant velocities ranging from $0.4,0.8,1.6,3.2$ to $6.4 \mu \mathrm{m} \mathrm{s}^{-1}$ for a dynamic force spectrum, otherwise and for titration experiments a standard velocity of $1.6 \mu \mathrm{m} \mathrm{s}^{-1}$ was used. After each curve acquired, the glass surface was moved horizontally by at least $100 \mathrm{~nm}$ to expose an unused, fresh surface spot. Typically, 50,000 - 100,000 curves were recorded per experiment. When quantitative comparisons of absolute forces were required, a single cantilever was used to move between multiple spatially separated spots to be probed on the same surface (created using the protocol described above). To calibrate cantilevers, the inverse optical cantilever sensitivity (InvOLS) was determined as the linear slope of the most probable value of typically 40 hard $(>2000 \mathrm{pN})$ indentation curves. Cantilevers spring constants were calculated using the equipartition theorem method with typical spring constants between $70-160 \mathrm{pN} \mathrm{nm}^{-150,51}$. A full list of calibrated spring constants from experiments presented in this work is provided in the supplementary methods, as the stiffness of the pulling handle, i.e., the cantilever, may influence the complex rupture and domain unfolding forces measured.

Calcium titration experiments. Buffer made from ultrapure water (resistivity $18.2 \mathrm{M} \Omega \mathrm{cm}$, arium pro, Sartorius, Goettingen, Germany), TRIS and $\mathrm{NaCl}$ (both Carl Roth, Karlsruhe, Germany) contained too much $\mathrm{Ca}^{2+}$ to reliably measure $\mathrm{Ca}^{2}$ + binding $\left(\mathrm{Ca}^{2+}{ }_{\text {-free buffer from ultrapure water already showed over } 50 \% \text { of }}\right.$ SdrG B1 domains in strong, $\mathrm{Ca}^{2+}$-bound state, even though the sample had been $\mathrm{Ca}^{2+}$ depleted with at least $10 \mathrm{mM}$ EDTA before). Instead, water (Ultra Quality HN68.2, Carl Roth, Karlsruhe, Germany) containing $\leq 10 \mathrm{ppt} \mathrm{Ca}^{2+}$ ( $\leq 10$ parts per trillion, i.e., $\leq 10 \mathrm{E}-12 \mathrm{~kg} \mathrm{~kg}^{-1}$, or $\leq 10 \mathrm{E}-9 \mathrm{~g} \mathrm{~L}^{-1}$, which computes to $\leq 0.250 \mathrm{nM}$ for $\mathrm{a} \mathrm{Ca}^{2+}$ ion) according to the manufacturer was used. Buffering was achieved by dissolving a $\mathrm{Ca}^{2+}$-free PBS tablet (Thermo Fisher Scientific, MA, USA). B domains immobilized on the surface were $\mathrm{Ca}^{2+}$ depleted with at least $10 \mathrm{mM}$ EDTA and after repeated rinsing in Ultra Quality $\mathrm{Ca}^{2+}$-free PBS, the titration was started. For each concentration, thousands of curves were acquired, before the surface was rinsed with the next $\mathrm{Ca}^{2+}$ concentration. As the discerning between strong and week state should not depend on cantilever stiffness, multiple experiment data using identical buffers were pooled to build statistics.

SMFS data analysis. Data analysis was carried out in Python 2.7 (Python Software Foundation $)^{52-54}$. Laser spot drift on the cantilever relative to the calibration curve was corrected via the baseline noise (determined as the last $5 \%$ of datapoints in each curve) for all curves and smoothed with a moving median (windowsize 300 curves). The InvOLS for each curve was corrected relative to the InvOLS value of the calibration curve.

Raw data were transformed from photodiode and piezo voltages into physical units with the cantilever calibration values: The piezo sensitivity, the InvOLS (scaled with the drift correction) and the cantilever spring constant (k).

The last rupture peak of every curve was coarsely detected and the subsequent $15 \mathrm{~nm}$ of the baseline force signal were averaged and used to determine the curve baseline, which was then set to zero force. The origin of molecule extension was then set as the first and closest point to zero force. A correction for cantilever bending, to convert extension data in the position of the cantilever tip was applied. Bending was determined through the forces measured and was used on all extension datapoints $(x)$ by correcting with their corresponding force datapoint $(F)$ as $x_{\text {corr }}=x-F / k$.

To detect unfolding or unbinding peaks, data were denoised with total variation denoising (raw, not denoised, data shown in plots) ${ }^{55,56}$, and rupture events detected as significant drops in force relative to the baseline noise. A three-regime polymer elasticity model by Livadaru et al. ${ }^{57}$ was used to model the behavior of contour lengths freed by unfolding events and transformed into contour length space ${ }^{58}$ (Livadaru model parameters were: stiff element $b=0.11 \mathrm{~nm}$ and bond angle $\gamma=41^{\circ}$ ). A quantum mechanical correction was used to account for peptide bond stretching at high forces ${ }^{59}$. Especially at forces larger than $1 \mathrm{nN}$, this correction was essential to be able to fit the data to polymer elasticity models 
accurately. Peaks were assigned their contour length in diagrams assembled through Kernel Density Estimates (KDE) of the contour length transformed forceextension data. The KDE bandwidth was chosen as $1 \mathrm{~nm}$. The loading rate was fitted as the linear slope of force vs. time of the last $4 \mathrm{~nm}$ preceding a peak.

For single $\mathrm{BE}$ model at a given force loading rate $\mathrm{r}$ (determined as most probable loading rate from all unfolding events through a KDE) with the parameters $\Delta \mathrm{x}$ and $\mathrm{k}_{\text {off, }, 0}$, the probability density $\mathrm{p}\left(F, r, \Delta x, k_{\text {off, }, 0}\right)$ to unfold at a given force $\mathrm{F}$ was fit to a normalized force histogram. For a superposition of two $\mathrm{BE}$ fits as in Fig. 4, the unfolding force histogram was fit with Eq. 1:

$$
\begin{aligned}
p_{\text {total }}\left(F, q, r_{1}, \Delta x_{1}, k_{\text {off }}{ }^{0}{ }_{1}, r_{2}, \Delta x_{2}, k_{\text {off }}{ }^{0}{ }_{2}\right)= & q \times p_{B E 1}\left(F, r_{1}, \Delta x_{1}, k_{\text {off }}{ }^{0}{ }_{1}\right) \\
& +(1-q) \times p_{B E 2}\left(F, r_{2}, \Delta x_{2}, k_{\text {off }}{ }^{0}{ }_{2}\right)
\end{aligned}
$$

Force loading rates $r_{1}$ and $r_{2}$ were assigned at a force $f_{\text {critical }}$ at the minimum value of $p_{\text {total }}$ between the maxima of both $\mathrm{BE}$ fits and then assigned to $\mathrm{BE} 1$ and $\mathrm{BE} 2$, as force loading rate and unfolding force correlate in a constant velocity experiment. The relative weight of each distribution was $q$ for BE1 and $(1-q)$ for BE2 with $0<q<1$.

For dynamic force, spectra rupture force histograms for the respective peaks and dynamic force spectra were assembled from all curves showing B domain unfolding, or (if applicable) a specific fingerprint domain, and/or a clean complex rupture event. The most probable loading rate of all complex rupture or domain unfolding events was determined with a KDE, bandwidth chosen through the Silverman estimator ${ }^{60}$. This value was used to fit the unfolding or rupture force histograms with the BE model for each pulling velocity ${ }^{61,62}$. Errors in all diagrams are given as the asymmetric full-width at half maximum (FWHM) of each probability distribution. A final fit was performed through the most probable rupture forces and loading rates for each pulling velocity to determine the distance to the transition state $\Delta \mathrm{x}_{0}$ and natural off-rate at zero force $k_{\text {off }}{ }^{0}$.

Homology models and simulations. Homology models for the SdrG B1, SdrG B2 domain, and SdrG N2N3-B1-B2 construct were created using Modeller 9.1963,64. Template file for the B domains was PDB 4JDZ (SdrD B1 domain) and for the SdrG N2N3-B1-B2 construct PDB 1R17 (SdrG N2N3) was added.

Model structures were equilibrated in water using the NAMD ${ }^{28}$ molecular dynamics package with setups created by $\mathrm{VMD}^{65}$ plugin QwikMD ${ }^{27}$. The CHARMM $36^{66}$ force field and TIP $3^{67}$ water model were used in all simulations. Structures were centered in a water box 15 Angstrom larger than the protein's longest dimension, $\mathrm{NaCl}$ was added to $150 \mathrm{mM}$. Minimization (2000 steps), then Annealing $(0.29 \mathrm{~ns}$, temperature rise $60 \mathrm{~K}$ to $300 \mathrm{~K}, 1 \mathrm{~atm}$ pressure, protein backbone restrained), then equilibration ( $1 \mathrm{~ns}$, temperature $300 \mathrm{~K}, 1 \mathrm{~atm}$ pressure, protein backbone restrained), then MD simulation (temperature $300 \mathrm{~K}, 1 \mathrm{~atm}$ pressure, no restraints) were performed in the $\mathrm{NpT}$ ensemble. Final simulations were run for at least $100 \mathrm{~ns}$ for individual B domains and at least $30 \mathrm{~ns}$ for SdrG N2N3-B1-B2.

Simulation parameters were: a distance cut-off of $12.0 \AA$ was applied to shortrange, non-bonded interactions, and $10.0 \AA$ for the smothering functions. Longrange electrostatic interactions were treated using the particle-mesh Ewald ${ }^{68}$ method. The pressure was maintained at $1 \mathrm{~atm}$ using Nosé-Hoover Langevin piston $^{69,70}$. The equations of motion were integrated using the reversible reference system propagator algorithm ( $\mathrm{r}$-RESPA) multiple time step scheme to update the short-range interactions every 1 steps and long-range electrostatics interactions every 2 steps. The time step of integration was chosen to be $2 \mathrm{fs}$ for all simulations. The temperature was maintained at $300 \mathrm{~K}$ using Langevin dynamics.

\section{Data availability}

Data supporting the findings of this manuscript are available from the corresponding authors upon reasonable request. A reporting summary for this article is available as a Supplementary Information file. The source data underlying Figs. 1e, f, $2 \mathrm{~b}-\mathrm{e}, 3 \mathrm{c}, \mathrm{d}$, and $4 \mathrm{c}-\mathrm{e}$ are provided as a Source Data file.

Received: 13 August 2018 Accepted: 17 October 2018

Published online: 12 November 2018

\section{References}

1. Foster, T. J., Geoghegan, J. A., Ganesh, V. K. \& Höök, M. Adhesion, invasion and evasion: the many functions of the surface proteins of Staphylococcus aureus. Nat. Rev. Microbiol. 12, 49-62 (2013).

2. Geoghegan, J. A. \& Dufrêne, Y. F. Mechanomicrobiology: how mechanical forces activate Staphylococcus aureus adhesion. Trends Microbiol. 26, 1-3 (2018).

3. Schwarz-Linek, U. et al. Pathogenic bacteria attach to human fibronectin through a tandem beta-zipper. Nature 423, 177-181 (2003).
4. Ganesh, V. K. et al. Structural and biochemical characterization of Staphylococcus aureus clumping factor B/ligand interactions. J. Biol. Chem. 286, 25963-25972 (2011)

5. Ponnuraj, K. et al. A "dock, lock, and latch" structural model for a Staphylococcal adhesin binding to fibrinogen. Cell 115, 217-228 (2003).

6. Bowden, M. G. et al. Evidence for the 'dock, lock, and latch' ligand binding mechanism of the staphylococcal microbial surface component recognizing adhesive matrix molecules (MSCRAMM) SdrG. J. Biol. Chem. 283, 638-647 (2008)

7. Herman, P. et al. The binding force of the staphylococcal adhesin SdrG is remarkably strong. Mol. Microbiol. 93, 356-368 (2014).

8. Vanzieleghem, T., Herman-Bausier, P., Dufrene, Y. F. \& Mahillon, J. Staphylococcus epidermidis affinity for fibrinogen-coated surfaces correlates with the abundance of the SdrG adhesin on the cell surface. Langmuir 31, 4713-4721 (2015).

9. Milles, L. F., Schulten, K., Gaub, H. E. \& Bernardi, R. C. Molecular mechanism of extreme mechanostability in a pathogen adhesin. Sci. (80-.). 359, 1527-1533 (2018).

10. Prystopiuk, V. et al. Mechanical forces guiding Staphylococcus aureus cellular invasion. ACS Nano Acsnano. 12, 3609-3622 (2018).

11. Herman-Bausier, P. et al. Staphylococcus aureus clumping factor A is a forcesensitive molecular switch that activates bacterial adhesion. Proc. Natl. Acad. Sci. USA 115, 5564-5569 (2018)

12. Vitry, P. et al. Force-induced strengthening of the interaction between Staphylococcus aureus clumping factor B and loricrin. mBio 8, e01748-17 (2017).

13. Grandbois, M. How strong is a covalent bond? Sci. (80-.). 283, 1727-1730 (1999).

14. Matsuoka, E. et al. Crystal structure of the functional region of Uro-adherence factor A from Staphylococcus saprophyticus reveals participation of the B domain in ligand binding. Protein Sci. 20, 406-416 (2011).

15. Hendrickx, A. Pa, Budzik, J. M., Oh, S.-Y. \& Schneewind, O. Architects at the bacterial surface - sortases and the assembly of pili with isopeptide bonds. Nat. Rev. Microbiol. 9, 166-176 (2011).

16. Ott, W., Jobst, M. A., Schoeler, C., Gaub, H. E. \& Nash, M. A. Single-molecule force spectroscopy on polyproteins and receptor-ligand complexes: the current toolbox. J. Struct. Biol. 197, 3-12 (2016).

17. Geoghegan, J. A., Foster, T. J., Speziale, P. \& Dufrêne, Y. F. Live-cell nanoscopy in antiadhesion therapy. Trends Microbiol. 25, 512-514 (2017).

18. Müller, D. J. \& Dufrêne, Y. F. Atomic force microscopy as a multifunctional molecular toolbox in nanobiotechnology. Nat. Nanotechnol. 3, 261-269 (2008).

19. Yu, H., Siewny, M. G. W., Edwards, D. T., Sanders, A. W. \& Perkins, T. T. Hidden dynamics in the unfolding of individual bacteriorhodopsin proteins. Sci. (80-.). 355, 945-950 (2017)

20. Höök, M. et al. The serine-aspartate repeat (Sdr) protein family in Staphylococcus epidermidis. Microbiology 146, 1535-1546 (2000).

21. Josefsson, E., O'Connell, D., Foster, T. J., Durussel, I. \& Cox, J. A. The binding of calcium to the B-repeat segment of SdrD, a cell surface protein of Staphylococcus aureus. J. Biol. Chem. 273, 31145-31152 (1998).

22. Josefsson, E. et al. Three new members of the serine-aspartate repeat protein multigene family of Staphylococcus aureus. Microbiology 144, 3387-3395 (1998).

23. Echelman, D. J. et al. CnaA domains in bacterial pili are efficient dissipaters of large mechanical shocks. Proc. Natl. Acad. Sci. 113, 2490-2495 (2016).

24. Valbuena, A. et al. On the remarkable mechanostability of scaffoldins and the mechanical clamp motif. Proc. Natl. Acad. Sci. 106, 13791-13796 (2009).

25. Verdorfer, T. et al. Combining in vitro and in silico single-molecule force spectroscopy to characterize and tune cellulosomal scaffoldin mechanics. $J$. Am. Chem. Soc. 139, 17841-17852 (2017).

26. Wang, X., Ge, J., Liu, B., Hu, Y. \& Yang, M. Structures of SdrD from Staphylococcus aureus reveal the molecular mechanism of how the cell surface receptors recognize their ligands. Protein Cell 4, 277-285 (2013)

27. Ribeiro, J. V. et al. QwikMD - integrative molecular dynamics toolkit for novices and experts. Sci. Rep. 6, 26536 (2016).

28. Phillips, J. C. et al. Scalable molecular dynamics with NAMD. J. Comput. Chem. 26, 1781-1802 (2005).

29. Lu, H. et al. Unfolding of titin immunoglobulin domains by steered molecular dynamics simulation. Biophys. J. 75, 662-671 (1998).

30. Stigler, J., Ziegler, F., Gieseke, A., Gebhardt, J. C. M. \& Rief, M. The complex folding network of single calmodulin molecules. Sci. (80-.). 334, 512-516 (2011).

31. Roman, A. Y. et al. Sequential binding of calcium ions to the B-repeat domain of SdrD from Staphylococcus aureus. Can. J. Microbiol. 62, 123-129 (2016).

32. Otten, M. et al. From genes to protein mechanics on a chip. Nat. Methods 11 $1127-1130$ (2014).

33. Walden, M. et al. An internal thioester in a pathogen surface protein mediates covalent host binding. eLife 4, 1-24 (2015). 
34. Linke-Winnebeck, C. et al. Structural model for covalent adhesion of the Streptococcus pyogenes pilus through a thioester bond. J. Biol. Chem. 289, 177-189 (2014).

35. Echelman, D. J., Lee, A. Q. \& Fernández, J. M. Mechanical forces regulate the reactivity of a thioester bond in a bacterial adhesin. J. Biol. Chem. M117, 777466 (2017).

36. Kang, H. J., Coulibaly, F., Clow, F., Proft, T. \& Baker, E. N. Stabilizing isopeptide bonds revealed in Gram-positive bacterial pilus structure. Sci. (80.). 318, 1625-1628 (2007).

37. Kang, H. J. \& Baker, E. N. Intramolecular isopeptide bonds: protein crosslinks built for stress? Trends Biochem. Sci. 36, 229-237 (2011).

38. Alegre-Cebollada, J., Badilla, C. L. \& Fernández, J. M. Isopeptide bonds block the mechanical extension of pili in pathogenic Streptococcus pyogenes. J. Biol. Chem. 285, 11235-11242 (2010).

39. Zakeri, B. et al. Peptide tag forming a rapid covalent bond to a protein, through engineering a bacterial adhesin. Proc. Natl. Acad. Sci. USA 109, E690-E697 (2012).

40. Zong, Y. et al. A 'Collagen Hug' model for Staphylococcus aureus CNA binding to collagen. EMBO J. 24, 4224-4236 (2005).

41. Deivanayagam, C. C. S. et al. Novel fold and assembly of the repetitive B region of the Staphylococcus aureus collagen-binding surface protein. Structure 8, 67-78 (2000).

42. Herman-Bausier, P. et al. Mechanical strength and inhibition of the Staphylococcus aureus collagen-binding protein Cna. mBio 7, e01529-16 (2016).

43. Moore, E. W. Ionized calcium in normal serum, ultrafiltrates, and whole blood determined by ion-exchange electrodes. J. Clin. Invest. 49, 318-334 (1970).

44. Fang, J. et al. Forced protein unfolding leads to highly elastic and tough protein hydrogels. Nat. Commun. 4, 2974 (2013).

45. Lv, S. et al. Designed biomaterials to mimic the mechanical properties of muscles. Nature 465, 69-73 (2010).

46. Beulin, Jemima, D. S. \& Ponnuraj, K. Steered molecular dynamics study reveals insights into the function of the repetitive B region of collagen- and fibrinogen-binding MSCRAMMs. J. Biomol. Struct. Dyn. 1102, 1-45 (2016).

47. Gibson, D. G. et al. Enzymatic assembly of DNA molecules up to several hundred kilobases. Nat. Methods 6, 343-345 (2009).

48. Studier, F. W. Protein production by auto-induction in high-density shaking cultures. Protein Expr. Purif. 41, 207-234 (2005).

49. Jobst, M. A., Schoeler, C., Malinowska, K. \& Nash, M. A. Investigating receptor-ligand systems of the cellulosome with AFM-based single-molecule force spectroscopy. J. Vis. Exp. 82, e50950 (2013).

50. Hutter, J. L. \& Bechhoefer, J. Calibration of atomic-force microscope tips. Rev. Sci. Instrum. 64, 1868-1873 (1993).

51. Butt, H. \& Jaschke, M. Calculation of thermal noise in atomic force microscopy. Nanotechnology 6, 1 (1995).

52. Hunter, J. D. Matplotlib: a 2D graphics environment. Comput. Sci. Eng. 9, 90-95 (2007).

53. Pedregosa, F. et al. Scikit-learn: machine learning in Python. J. Mach. Learn. Res. 12, 2825-2830 (2011).

54. van der Walt, S., Colbert, S. C. \& Varoquaux, G. The NumPy array: a structure for efficient numerical computation. Comput. Sci. Eng. 13, 22-30 (2011).

55. Condat, L. A direct algorithm for 1-D total variation denoising. IEEE Signal Process. Lett. 20, 1054-1057 (2013).

56. Rudin, L., Osher, S. \& Fatemi, E. Nonlinear total variation based noise removal algorithms. Phys. D. Nonlinear Phenom. 60, 259-268 (1992).

57. Livadaru, L., Netz, R. R. \& Kreuzer, H. J. Stretching response of discrete semiflexible polymers. Macromolecules 36, 3732-3744 (2003).

58. Puchner, E. M., Franzen, G., Gautel, M. \& Gaub, H. E. Comparing proteins by their unfolding pattern. Biophys. J. 95, 426-434 (2008).

59. Hugel, T., Rief, M., Seitz, M., Gaub, H. \& Netz, R. Highly stretched single polymers: atomic-force-microscope experiments versus Ab-Initio theory. Phys. Rev. Lett. 94, 048301 (2005).

60. Silverman, B. Density estimation for statistics and data analysis. Monogr. Stat. Appl. Probab. 37, 1-22 (1986).

61. Izrailev, S., Stepaniants, S., Balsera, M., Oono, Y. \& Schulten, K. Molecular dynamics study of unbinding of the avidin-biotin complex. Biophys. J. 72, 1568-1581 (1997).
62. Evans, E. \& Ritchie, K. Dynamic strength of molecular adhesion bonds. Biophys. J. 72, 1541-1555 (1997).

63. Šali, A. \& Blundell, T. L. Comparative protein modelling by satisfaction of spatial restraints. J. Mol. Biol. 234, 779-815 (1993).

64. Webb, B. \& Sali, A. Comparative protein structure modeling using MODELLER. Curr. Protoc. Bioinforma. 47, 5.6.1-5.6.32 (2014).

65. Humphrey, W., Dalke, A. \& Schulten, K. VMD: visual molecular dynamics. J. Mol. Graph. 14, 33-38 (1996).

66. Best, R. B. et al. Optimization of the additive CHARMM all-atom protein force field targeting improved sampling of the backbone phi, psi and sidechain chi(1) and chi(2) dihedral angles. J. Chem. Theory Comput. 8, 3257-3273 (2012).

67. Jorgensen, W. L., Chandrasekhar, J., Madura, J. D., Impey, R. W. \& Klein, M L. Comparison of simple potential functions for simulating liquid water. $J$. Chem. Phys. 79, 926 (1983).

68. Darden, T., York, D. \& Pedersen, L. Particle mesh Ewald: an N.log(N) method for Ewald sums in large systems. J. Chem. Phys. 98, 10089 (1993).

69. Feller, S. E., Zhang, Y., Pastor, R. W. \& Brooks, B. R. Constant pressure molecular dynamics simulation: the Langevin piston method. J. Chem. Phys. 103, 4613-4621 (1995).

70. Martyna, G. J., Tobias, D. J. \& Klein, M. L. Constant pressure molecular dynamics algorithms. J. Chem. Phys. 101, 4177-4189 (1994).

\section{Acknowledgements}

We gratefully acknowledge funding from the Deutsche Forschungsgemeinschaft DFG (SFB1032, GA309/11). We thank Ellis Durner, Markus A. Jobst, Wolfgang Ott, and Tobias Verdorfer for work on instrumentation and/or surface chemistry; Rafael C. Bernardi for pointers concerning modeling.

\section{Author contributions}

H.E.G. and L.F.M. designed the research. T.N. cloned and expressed constructs, and prepared surface chemistry reagents. E.M.U. and L.F.M performed and analyzed experiments. H.E.G. and L.F.M. wrote the manuscript.

\section{Additional information}

Supplementary Information accompanies this paper at https://doi.org/10.1038/s41467 018-07145-6.

Competing interests: The authors declare no competing interests.

Reprints and permission information is available online at http://npg.nature.com/ reprintsandpermissions/

Publisher's note: Springer Nature remains neutral with regard to jurisdictional claims in published maps and institutional affiliations.

Open Access This article is licensed under a Creative Commons Attribution 4.0 International License, which permits use, sharing, adaptation, distribution and reproduction in any medium or format, as long as you give appropriate credit to the original author(s) and the source, provide a link to the Creative Commons license, and indicate if changes were made. The images or other third party material in this article are included in the article's Creative Commons license, unless indicated otherwise in a credit line to the material. If material is not included in the article's Creative Commons license and your intended use is not permitted by statutory regulation or exceeds the permitted use, you will need to obtain permission directly from the copyright holder. To view a copy of this license, visit http://creativecommons.org/ licenses/by/4.0/

(C) The Author(s) 2018 